\title{
SHORT-TERM AND LONG-TERM STABILITY IN ELECTRONIC COMMUNICATION NETWORKS
}

\author{
ERIC QUINTANE \\ Institute of Management \\ University of Lugano \\ Lugano, 6900, Switzerland \\ And \\ School of Management \\ Universidad de los Andes \\ Bogota, Colombia \\ PHILIPPA E. PATTISON \\ The University of Melbourne \\ GARRY L. ROBINS \\ The University of Melbourne \\ JOERI M. MOL \\ The University of Melbourne
}

\begin{abstract}
Network researchers typically focus on patterns of stable relationships, where stability represents the unfolding of social processes over long time frames. By contrast, we argue and empirically demonstrate that social interactions exhibit regularities across different time frames (short and long-term), reflecting distinct social processes.
\end{abstract}

\section{INTRODUCTION}

In this paper, we examine the temporal nature of social processes, focusing on the difference between short- and long-term stability. Rather than referring to a static picture of a set of social relations, social stability should be conceived as representing the regularities in the unfolding of social processes within a certain time frame. Hence stability is inherently bound by time. The extant literature, however, largely ignores specific time frames within which stable patterns of social interactions can occur. In doing so, it implicitly assumes that long-term time frames - usually of indefinite length - capture the essence of network structures and network dynamics. By contrast, we argue that regularities in network exchanges also occur across shorter time frames and carry significance above and beyond long-term exchange patterns. Social processes - even of the same type - have different meanings depending on whether they operate in the short- or long-term.

We illustrate the relevance of distinguishing between short- and long-term processes in the context of project teams, where we measure the occurrence of reciprocity and closure in these two time frames. We argue that long-term reciprocity and closure represent team cohesion, while their short-term expressions represent a team's adaptation to change. More specifically, we investigate how project teams balance the demands of maintaining overall team cohesion in the 
face of constant compositional change with having to adapt to short-term contingencies.

Using Butts' (2008) Relational Events Framework and a unique e-mail dataset, we examine the existence of regularities in reciprocity and closure across different time frames for two teams whose e-mail communications we monitored during approximately a six month period. We show that above and beyond the influence of formal structure, homophily, and longterm stable processes, reciprocity and closure exhibit stability in the short-term. Furthermore, our results show that only the high-performing team was able to maintain cohesion in the face of constant compositional change, indicated by stable patterns of reciprocity and closure in interaction patterns in both the short- and the long-term.

A key contribution of our paper is to show that the dynamics of teamwork cannot be assumed to be captured by long-term patterns of interactions alone. So, while team performance has been shown to depend on features of network structures that are stable in the long-term (e.g., Balkundi and Harrison, 2006; Cummings and Cross, 2003; Sparrowe et al., 2001), it may also be determined by the capacity of team members to establish stability for various social processes across different time frames.

\section{THEORETICAL BACKGROUND}

Current organizational network studies often consider past structural configurations as homogenous and stable across long time frames, typically of indefinite duration, and do not consider the various time frames of social life (i.e., from short-term to long-term). A major reason why a short-term time frame is usually discounted in network studies is the difficulty of accessing reliable information about shorter-term temporal patterns. Another reason is that shortterm interactions are often considered as volatile and merely adding noise to long-term trends in relationships. Finally, there may also be an implicit assumption that short-term time frames can be safely dismissed, not just because of their inherent volatility, but also because regularities that exist in the short-term are seen to converge with the regularities pertaining to the long-term.

We understand a social network as the "pattern of regular exchange connections that actors recognize as important to their activity" (Rank et al., 2010, p. 745). We therefore do not presuppose that regularities in exchange connections can only be reliably understood from a long-term perspective and we believe there is good reason to investigate these regularities from a temporal perspective that allows for varying timeframes across which social dynamics take place. Our approach is not to compare short-term interactions with long-term reported network ties, as has been done previously in the literature (e.g., Quintane \& Kleinbaum, 2011). Rather, we contrast interaction patterns in the short- and long-term, arguing that the long-term patterns are indicators of stable social relationships, and hence of network ties, while short-term patterns are more ephemeral interaction structures arising from particular coordinated tasks. We incorporate two key dimensions of social relations: their interactional foundations and the temporal dimensions on which they are based. In other words, we are concerned with change in social structure as captured by sociotemporal patterns within sequences of interactions.

We investigate the dynamics of internal network structure of teams, focusing on the temporal expression of these dynamics in the short- and long-term. Teams are especially suitable for this study because of the salience of temporal aspects of social relations. Also, while teams are a basic unit of collaboration in organizations, the organizational network literature only features a few studies that investigate the dynamics of internal network structures of teams (Katz et al., 2004). 
A specific aspect of project teams that affects social relationships and internal network structure is compositional change. Compositional change in project teams refers to team members entering and exiting the team as their skills are needed to complete certain tasks at a certain point in time (Summers et al., 2011). Compositional change implies that team members have fewer opportunities to develop and maintain stable inter-personal relationships, which can have a negative effect on team cohesion (van der Vegt et al., 2010). Cohesion, or the inclination of group members to forge social bonds, has been one of the more thoroughly researched emergent states in team research and results show that cohesion is associated with positive performance (Casey-Campbell and Martens, 2009; Mathieu et al., 2008). Compositional change in project teams complicates the development of cohesion through the disruption of interpersonal relationships. Clearly, developing strong cohesiveness with constantly changing group membership is an arduous task.

In order to explore the extent to which a project team is cohesive over time we focus on two concepts that have been used in previous research as indicators of cohesiveness: reciprocity and closure. Reciprocity refers to the extent to which an interaction that is directed from employee A to employee B potentiates interaction directed from B to A. In an organizational and team context, a stable pattern of reciprocated relationships is considered to indicate the existence of social cohesion and trust between team members (Friedkin, 2004). However, we do not expect all reciprocal interactions in dyads to lead to a long-term reciprocal relationship. A short-term pattern of reciprocity indicates that interactions between two individuals were sustained and reciprocal for a short duration, but not necessarily sustained over a longer period of time. In other words, short-term reciprocal interactions may not necessarily lead to the existence of a long-term relationship.

The process of closure, i.e., the emergence of a relationship between an individual's tie partners, has been highlighted as a key mechanism in the building of social structure (Burt, 1992; Coleman, 1988; Granovetter, 1982; Uzzi, 1996, 1997). The existence of closure can be considered as an indicator of cohesiveness (Friedkin, 2004; Granovetter, 1973). Groups that exhibit more closure are argued to be denser, with redundant pathways for information exchange, and prone to the development of group norms (Burt, 1992). Within an organizational and group context, we argue that closure can also be representative of the need of individuals to exchange information to coordinate their activities for specific tasks when more than two employees are concerned. As such, we consider that closure can reflect the more time-sensitive coordination requirements of an activity and expect that closure can be activated across short time frames of specific duration in order to respond to the immediate demands of a specific task, as well as being representative of a process of longer-term cohesion within the team.

\section{EMPIRICAL SETTING}

In order to demonstrate the relevance and distinctiveness of regularities in sequences of interactions over different time frames, we examine two separate teams in a medium sized, project-based digital marketing agency. The two teams were engaged in the development of electronic platforms for the promotion of products and events for a single global client. We obtained a complete record of the e-mail communication patterns within the two teams throughout the duration of their respective projects ( 5 and 7 months). Both projects had a high degree of task complexity, they were tendered and contracted by the same client organization, had a similar number of employees involved (30\% of which were involved in both projects), 
required the same skill sets for completing similar tasks, were roughly of the same scale in terms of budget and time duration, occurred at the same period (between January and July 2008) and both involved high levels of knowledge intensity. The project managers of both teams were freelancers. One project was successful and the other unsuccessful.

Besides e-mail communication and basic demographic information, we obtained access to information relating to each project, most notably the time employees spent on their projects on a weekly basis. We combined employee's time sheets with the information about employees' involvement in their project and created a matrix reflecting the evolving composition of each project team over time. From the original dataset containing all the electronic communications of the company, we extracted two datasets (one per project). For each project, we identified the earliest and latest weeks with hours charged to the project for each employee - these are assumed to define the period of the person's involvement. Sender-recipient pairs were then filtered out unless the e-mail was sent in a week covered by the period of involvement of both the sender and the recipient. We further reduced these two datasets by retaining only e-mails that have less than four recipients (see Kossinets and Watts, 2006). The final dataset for Project A contains 10,320 time stamped dyadic communications and 6,925 dyadic communications for project B.

We applied Butts' (2008) Relational Event Framework to investigate the communication activities undertaken by team members (see Quintane et al. 2012, for details of the preparation of the dataset and estimation of the model). Briefly, the model is equivalent to a multinomial conditional logistic regression. Each event in the sequence is a dependent variable and for each real event we create a risk set of potential events that could have occurred at the same time as the real event using the employees actively working on the project on a given week.

\section{Variables and Controls}

We created statistics to represent the sociotemporal interaction patterns of interest: specifically, reciprocity and closure at each of two different time scales. For reciprocity, as the relational event that we predicted was the presence of an e-mail interaction between the sender and the recipient, we created a measure capturing the proportion of previous e-mails from the recipient to the sender out of all the e-mails sent by the recipient. A positive effect indicated a propensity for prior reciprocal events (e-mails from the recipient to the sender) within the designated time period to potentiate an e-mail communication (from the sender to the recipient) while a negative effect indicated a tendency for prior reciprocal events to be associated with a reduced probability of an e-mail communication. Our statistics for closure captured the extent to which a communication was potentiated by prior communications from the sender to third parties and from the same third parties to the recipient as a proportion of the total communications that were sent by the sender and received by the recipient. Combined with the relational event, a positive effect in the statistic indicated a propensity for individuals who had a large proportion of their total communications with common third party(ies) to also communicate together directly. A negative effect indicated that these individuals tended not to communicate together. All the statistics were standardized to make their effects more comparable.

We invoked time frames that range from short-term to long-term. To understand the impact of the recent past on future communication behavior, we selected a threshold of 24 hours. The inclusion of the month as a second time frame enabled us to capture patterns of activity that were embedded into longer time frames. The same statistics were computed for each time frame. 
This implied that the time frames were nested (i.e. a sequence of actions that happened in the short-term also happened in the long-term). The model developed here is sensitive to each potential form of regularity in each time frame, and therefore enabled us to assess the effect of the potential regularity across short- and long-term periods, with each effect estimated taking account of the other fitted effects.

In order to understand the existence of regularities in different temporal dimensions for the interaction patterns described above, we controlled for several relevant attributes of the actors, providing information about their backgrounds and organizational roles as well as density and degree indicators.

\section{RESULTS AND DISCUSSION}

Our results (Table available upon request from the authors) showed significant effects of reciprocity and closure on a daily basis, highlighting the existence of regular patterns of social interaction over shorter time frames. The strong short-term focus of reciprocity is in line with our prediction that e-mail replies and reciprocation more generally are expected within a short time frame given the dynamic and time-driven environment of these project teams. The persistence of this effect over longer time frames suggests that team members also develop more sustained and reciprocal relationships. Even more striking is the strong short-term tendency for closure, to the extent that a long-term pattern for closure is absent in one of the two teams. We see the strong propensity for closure in the short-term as a sign that team members have to alternately activate and deactivate complex coordination structures within short time frames in order to respond to the demands of the tasks, of the client, or of the environment more generally. The time frame of these activations/deactivations is very informative of the dynamic nature of the projects in which collaborations are formed within a day. The addition of a significant monthly pattern in Team A also means that certain collaboration structures are stable over the long-term and therefore may provide evidence of the continuous collaboration between a selected number of core team members over the life of the project.

Additional qualitative information about Team B could shed light on the absence of a significant long-term closure pattern. Interviews with the Head of Project Management indicated that the team failed to develop a "cohesive team spirit" throughout their work on the project and that there was little sense of continuity and belonging felt by the team members. These comments are consistent with the argument that closure is an important structural indicator of team cohesiveness, and that longer-term closure may indicate cohesiveness that has become a norm that extends beyond the immediate exigencies of a team dealing with a specific issue.

Hence, a possible interpretation of these patterns of closure is that Team A may have tended to be more cohesive over the course of the project, potentially focused on a core group, coupled with a short-term closure effect that enabled the completion of tight deadlines while meeting the contingent requirements inherent to the nature of the work of the team. By contrast, Team B was confronted with a series of ad-hoc requirements for closure, yet the absence of a longer-term significant effect suggests that a cohesive structure failed to emerge over time and thus may explain the team's lack of overall cohesiveness. It is only by considering these different temporal periods that we are able to see a specific structural correlate that may be indicative of the different performance levels of the two teams. 
The main finding of our investigation is the empirical demonstration of the existence of short-term stability in the form of regular patterns of communication within 24-hour periods that are distinct from, and not subsumed by, longer-term patterns. We found regular patterns of social interactions that are significant in the short-term above and beyond indicators of formal structure, generic tendencies towards homophily, changes in group composition, and long-term patterns of interaction. This result underlines the importance of understanding social interactions in terms of distinct time frames and supports the characterization of these regularities as sociotemporal in nature, since the impact of each effect is temporally bounded. The assumption that all regularities in shorter time frames are absorbed into longer-term regularities is implausible in light of our results. We see clear signs of regular interaction patterns that exist in shorter time frames beyond or more strongly than those that exist in longer time frames. In order to further nuance our understanding of the concept of stability, we emphasize the notion of regularity. Our results do not simply show that interactions occur in the short-term. They show that interactions occur regularly and repetitively within time frames of short duration and that these effects exist above and beyond (i.e. they are distinct from and in addition to) those regularities captured within time frames with longer duration.

\section{CONCLUSIONS}

This research responds to various calls for additional investigation of group dynamics (see Cronin et al., 2011). Our results show that in the context of project teams, a single focus on long-term patterns of social interactions is not sufficient to capture the full extent of the dynamics of teamwork. While stable long-term structure of advice and friendship clearly have an influence on team performance (Balkundi and Harrison, 2006; Cummings and Cross, 2003; Sparrowe et al., 2001), our results suggest that investigating communication structures - and by extension task coordination in project teams - requires an examination of the temporal dimensions of the social structure of the team, directing specific attention to short-term patterns. The interaction structure of teams can be considered as having regular expressions over a range of time frames (from short-term to long-term) and understanding how specific regularities in the sequences of interactions are embedded within different time frames may prove instrumental in understanding performance differentials of teams. For example, a team in which most patterns of interaction are rooted in the long-term could be very stable, but may have little potential for change and adaptation because regularities in communications would follow a set of patterns that are repeated continuously over time. By contrast, a team in which most patterns of interaction are expressed in the short-term may have difficulties to retain stability over time, which may be a cause of internal conflicts and team dissolution. Further research in the temporal dimension of team network structure is required to provide additional insights into these issues.

\section{REFERENCES AVAILABLE FROM THE AUTHOR(S)}




\section{University Library}

\section{- M M N E R VA A gateway to Melbourne's research publications}

Minerva Access is the Institutional Repository of The University of Melbourne

Author/s:

Quintane, E;Pattison, PE;Robins, GL;Mol, JM

Title:

Short-term and long-term stability in electronic communication networks

Date:

2013-01-01

Citation:

Quintane, E., Pattison, P. E., Robins, G. L. \& Mol, J. M. (2013). Short-term and longterm stability in electronic communication networks. Academy of Management 2013 Annual Meeting, AOM 2013, 2013, (1), pp.361-366. Academy of Management. https:// doi.org/10.5465/AMBPP.2013.45.

Persistent Link:

http://hdl.handle.net/11343/247847 